\title{
Corrigendum
}

\section{Automated detection of genetic abnormalities combined with cytology in sputum is a sensitive predictor of lung cancer}

Ruth L Katz, Tanweer M Zaidi, Ricardo L Fernandez, Jingpin Zhang, Weigong He, Charisse Acosta, Michal Daniely, Lea Madi, Mary A Vargas, Qiong Dong, Xiaoying Gao Feng Jiang, Nancy P Caraway, Ara A Vaporciyan, Jack A Roth and Margaret R Spitz

Modern Pathology (2008) 21, 1065; doi:10.1038/modpathol.2008.126

Correction to: Modern Pathology advance online publication 23 May 2008; doi: 10.1038/ modpathol.2008.71; Modern Pathology (2008) 21, 950-960; doi:10.1038/modpathol.2008.71
In this article, two authors' names were listed incorrectly. The correct author names are Xiaoying Gao and Feng Jiang. 